\title{
IMAGE ANALYSIS \& STEREOLOGY: 2017 RESEARCH HIGHLIGHTS
}

\author{
TOMAŽ VRTOVEC
}

University of Ljubljana, Faculty of Electrical Engineering, Tržaška cesta 25, SI-1000 Ljubljana, Slovenia e-mail: tomaz.vrtovec@fe.uni-lj.si

\begin{abstract}
A detailed overview of the 22 contributions, published in volume 36 (2017) of Image Analysis \& Stereology (IAS), is presented. Most of the contributions are relatively interdisciplinary, however, they can be assigned to the following fields of study: computer vision (2), image analysis (2), materials science (3), medical imaging (3), stereology (8) and stohastic geometry (2). In addition, two editorials were published, while four of the contributions in the field of stereology are review papers for the special topic "The History of Stereology". It can be concluded that the readership was offered with a large variety of topics within the broader multidisciplinary field of stereology and image analysis, therefore reflecting the scope of IAS.
\end{abstract}

Keywords: computer vision, image analysis, materials science, medical imaging, research highlights, stereology, stohastic geometry.

\section{INTRODUCTION}

Image Analysis \& Stereology, volume 36 (2017), consisted of three issues that published a total of 22 contributions. Among these, 15 were original research papers, one technical report, four review papers and two editorials. Although most of the contributions are relatively interdisciplinary, they can be approximately attributed to the following six fields of study, listed in alphabetical order: computer vision, image analysis, materials science, medical imaging, stereology and stohastic geometry. An overview of the contributions with the corresponding fields of study is provided in Table 1. According to the overview of the IAS research highlights in volume 35 (2016) by the IAS editor-in-chief (Kreft, 2017), in 2017 an increase of contributions in comparison to the year before was noticed (i.e., 22 vs. 16), however, the number of original research papers was approximately the same (i.e., 16 vs. 15), as all four review papers are part of the special topic entitled "The History of Stereology" that was published in issue 3 and guest edited by Dr. Aneta Gądek-Moszczak from the Cracow University of Technology, Poland. In the following sections, a detailed overview of the contributions of IAS volume 36 (2017) is presented.

\section{RESEARCH HIGHLIGHTS}

\section{COMPUTER VISION}

As a computer vision application, Kartbaev et al. (2017) proposed an approach to the implementation of an identity authentication system based on image face recognition that, besides comparing the image with reference samples, takes into account also the change of face features over time (e.g., due to aging). For the purpose of increasing the efficiency of automated face recognition, the proposed approach is based on the adaptive neuro-fuzzy inference system (ANFIS), a special type of a fuzzy logic inference that is combined with an artificial neural network (AAN) architecture for supervised learning that reduces the rate of errors in the determination of rules in fuzzy logic. The proposed implementation has a three-tier architecture consisting of preliminary processing, recognition and identification of images obtained from monitoring. The fuzzy base of products is based on expert knowledge, and allows the assessment of possible changes in key characteristics that are used for image-based identity authentication. As the performed experiments showed a relatively high efficiency with a low value of learning errors, the proposed implementation was recommended for a practical application that would allow to predict changes in individuals over time, and in general improve the authentication accuracy and efficiency.

Another growing application of computer vision is the development of advanced driver-assistance systems (ADAS), which provide automated or enhanced features to the vehicles for improving the safety and driving experience, such as collision avoidance, maneuvering assistance or safeguarding alerts. One of the increasingly popular ADAS is the surround view system (SVS), or omniview technology, which is a solution based on computer vision that generates a $360^{\circ}$ view of the vehicle surroundings through mounted cameras and image processing algorithms. As an element of SVS, Bawa et al. (2017) proposed a novel algorithm for fast and computationally efficient transformation of input images captured by four 
Table 1 . An overview of the contributions to volume 36 (2017) of Image Analysis \& Stereology according to the field of study, listed in alphabetical order (asterisks indicate the contributions to the special topic entitled "The History of Stereology", published in issue 3).

\begin{tabular}{|c|c|c|c|c|}
\hline Field & Quantity & Author(s) & Issue & Pages \\
\hline \multirow[t]{2}{*}{ (Editorial) } & \multirow[t]{2}{*}{2} & Kreft & 1 & $1-3$ \\
\hline & & Gądek-Moszczak* & 3 & $151-152$ \\
\hline \multirow[t]{2}{*}{ Computer vision } & \multirow[t]{2}{*}{2} & Kartbaev et al. & 1 & $51-64$ \\
\hline & & Bawa et al. & 2 & $141-150$ \\
\hline \multirow[t]{2}{*}{ Image analysis } & \multirow[t]{2}{*}{2} & Koçak & 1 & $15-23$ \\
\hline & & Baran and Tabor & 3 & $223-232$ \\
\hline \multirow[t]{3}{*}{ Materials science } & \multirow[t]{3}{*}{3} & Šedivý et al. & 1 & $5-13$ \\
\hline & & Jouannot-Chesney et al. & 2 & $95-103$ \\
\hline & & Wejrzanowski et al. & 2 & $105-110$ \\
\hline \multirow[t]{3}{*}{ Medical imaging } & \multirow[t]{3}{*}{3} & Niedźwiecki et al. & 1 & $35-41$ \\
\hline & & Khordehchi et al. & 2 & $65-78$ \\
\hline & & Nieniewski and Zajączkowski & 2 & 79-94 \\
\hline \multirow[t]{8}{*}{ Stereology } & \multirow[t]{8}{*}{8} & Li et al. & 2 & $111-120$ \\
\hline & & González-Villa et al. & 2 & $121-132$ \\
\hline & & Kiderlen and Dorph-Petersen & 2 & 133-139 \\
\hline & & Gual-Arnau et al. & 3 & $233-241$ \\
\hline & & Cruz-Orive* & 3 & $153-177$ \\
\hline & & Vedel Jensen and Gundersen ${ }^{*}$ & 3 & $179-185$ \\
\hline & & Kubínová et al. ${ }^{*}$ & 3 & $187-205$ \\
\hline & & Gądek-Moszczak and Matusiewicz* & 3 & $207-221$ \\
\hline \multirow[t]{2}{*}{ Stohastic geometry } & \multirow[t]{2}{*}{2} & Beneš et al. & 1 & $25-33$ \\
\hline & & Rønn-Nielsen et al. & 1 & $43-49$ \\
\hline
\end{tabular}

vehicle-mounted cameras with fish-eye lenses into the required top-down view, irrespective of the camera tilt angle. After estimating the center of distortion, the proposed algorithm corrects the fish-eye images into rectilinear images by radial distortion correction and pixel stretching, and then generates a top view perspective from the resulting images by applying a homography technique to account for the changes of the camera projection plane. The proposed algorithm was shown to produce satisfactory results on random images independent of the camera tilt angle while keeping the computation and hardware costs to a minimum.

\section{IMAGE ANALYSIS}

Steganography is concerned with concealing a file, message, image or video within another file, message, image or video, therefore dealing with important aspects of image analysis. Koçak (2017) proposed a combined cryptographic and steganographic technique called couple layered security model (CLSM) for the purpose of concealing a secret message within an image without its visual tampering. The text message is first encrypted with the symmetric-key one-time pad algorithm using a key of 128-bit maximal length, and then embedded into a color image with a 4-bit substitution on the least significant bits (LSB) of the green and blue channels to account for the spectral sensitivity of the human eye. The performance of CLSM was evaluated in terms of the peak signalto-noise ratio that measures the quality between the original and tampered image, and structural similarity index that predicts the perceived quality of the tampered image. The results revealed that CLSM provides a relatively good image quality in return for a higher capacity of the secret information that is embedded into the image.

A more general topic of image analysis was studied by Baran and Tabor (2017), who proposed a novel algorithm for object boundary delineation based on principal geodesic analysis (PGA) for the purpose of object detection and segmentation. By applying 
PGA, a statistical shape model of the object of interest is first trained from angular representations of automatically placed and uniformly distant landmarks, and then used to detect object boundaries in a previously unseen image by iterative elongation of a partial boundary along the oversegmented image, obtained by a superpixel segmentation algorithm (e.g., watershed). The proposed algorithm was evaluated on two datasets of X-ray images of the knee and foot, and two datasets of synthetically generated images of stars, triangles and squares obscured with noise. The results for object detection and segmentation indicated that the proposed algorithm allows for a very efficient shape representation using angles without the need of boundary initialization for the shape matching process.

\section{MATERIALS SCIENCE}

Understanding the structural mechanisms of alloys is an important topic within materials science, and from this perspective Šedivý et al. (2017) focused on the three-dimensional (3D) morphology of aluminum alloy grains using parametric ellipsoid-based tessellation models. In comparison to voxel-based alternatives, tessellation models offer a substantial reduction in the storage space required for large 3D data describing polycrystalline microstructures, moreover, ellipsoid-based models enable an easier derivation of grain size and shape characteristics. The authors computed structural geometrical features for two aluminum alloy samples, $\mathrm{Al}-3 \mathrm{wt} \% \mathrm{Mg}$ $0.2 \mathrm{wt} \% \mathrm{Sc}$ and $\mathrm{Al}-1 \mathrm{wt} \% \mathrm{Mg}$, and acquired 3D images of their microstructure respectively by electron backscatter diffraction from a scanning electron microscope and by 3D X-ray diffraction microscopy. Finally, they compared their tessellation results to empirical data, and obtained a relatively good fit in terms of correctly assigned voxels as well as grain volume and surface boundary. Moreover, they showed that the structural geometrical features, such as the dihedral angle and Gaussian curvature, can be consistently obtained.

On the other hand, Jouannot-Chesney et al. (2017) explored the relationship between the percolation threshold of a structure, a mathematical concept that indicates the formation of long-range connectivity in random systems, and its Euler-Poincaré characteristic (EPC), which describes the topological shape of a structure. The authors performed simulations on a number of random structures with increasing densities in two-dimensional and 3D space, and for each structure model, the percolation threshold was compared with a number of typical points, e.g., extrema, zero crossings and inflection points. They concluded that the percolation threshold is generally not related to any singular point of the curves reflecting the evolution of the structure EPC, and that therefore a direct link between the percolation threshold of a structure and its topology does not exist.

Numerical modeling was also applied by Wejrzanowski et al. (2017), who focused on openporous materials that are being increasingly used for manufacturing different products, such as energy absorbers, filters, bone implants, catalysts and heat exchangers. Specifically, they presented a model for open-cell foams based on the Laguerre-Voronoi tessellation and a model for open-porosity tapes based on the sphere representation, that were respectively tested on samples of a commercially available aluminum foam and a casted molten carbonate fuel cell. Micro-computed tomography images of the samples were acquired, and parameters such as porosity, specific surface area, mean pore size and mean strut size were extracted using a dedicated software for the purpose of characterizing the porous structure of samples. These parameters were then compared to corresponding geometrical features of the proposed models, showing that both models are good representations of the studied open-porous materials, as they exhibited a negligibly small discrepancy against the studied samples, observed through the agreement of the measured parameters. The authors concluded that the proposed models are representative and can be applied for further research.

\section{MEDICAL IMAGING}

In radiotherapy as part of cancer treatment using ionizing radiation delivered by a linear accelerator device, the mechanical isocenter refers to the point in space about which various components of the treatment device rotate. On the other hand, the radiation isocenter refers to the point in space where radiation beams intersect when the gantry is rotated, and its geometrical localization affects tumor targeting and therefore the overall quality of the treatment. Ideally, the mechanical and radiation isocenter coincide, however, due to inaccuracies in the device construction and weight of its components they may move in space. The Winston-Lutz test is commonly used for estimating the isocentric accuracy of radiotherapy devices, where a small radio-opaque sphere (i.e., a ball marker) is positioned at the isocenter, and then a series of images are acquired at different gantry orientations to measure the offset of the sphere relative to the radiation field center. Niedźwiecki et al. (2017) designed a new automated tool based on image analysis to support the isocenter quality assurance by extracting additional parameters from the Winston-Lutz test images of a cube phantom. 
They showed that the gantry rotation angle as well as the ratio of the distance from the detector panel and the marker to the radiation source can be determined by imaging such a basic cube phantom, which was supported by a very good agreement between the nominal and estimated values.

One of the applications of radiotherapy is for the treatment of lung cancer, however, most patients are diagnosed when presenting symptoms with the disease already in an advanced stage. To reduce the mortality rate from lung cancer, early diagnosis is therefore of utmost importance. The detection and interpretation of lung nodules in their early stage of growth is fundamental to the diagnosis of lung cancer, and Khordehchi et al. (2017) proposed a novel framework for computer-aided detection of lung nodules from computed tomography (CT) images. In the preprocessing step, each CT image crosssection is first smoothed by an adaptive diffusionbased algorithm with nonlinear partial differential equations, and then a combination of morphological filtering operations is used to detect the edges and extract the regions of interest (ROIs). Afterwards, the CT cross-section is segmented by applying the statistical region merging algorithm to the extracted ROIs, and the obtained segments are, according to an intersection criterion, connected together in consecutive cross-sections to form three-dimensional objects representing nodule candidates. For each nodule candidate, six features (four intensity-based, one shape-based and one texture-based) are extracted from the corresponding image volume, and finally used to classify the candidates into nodules and non-nodules using support vector machines. The proposed framework was evaluated on two publicly available CT image datasets with reference lung nodule annotations, namely the Early Lung Cancer Action Program (ELCAP) public lung image database and the Lung Image Database Consortium (LIDC) image collection, and against nine alternative state-ofthe-art methods. The results showed that the proposed framework achieved an average classification accuracy of $90 \%$ for the ELCAP and $95 \%$ for the LIDC dataset. In comparison to alternative methods, the proposed framework was ranked first in terms of classification sensitivity on both datasets, while in terms of specificity and accuracy it was ranked above average. Moreover, two clinical experts also validated the results and confirmed the efficiency of the proposed framework.

Another study related to diagnostic imaging was presented by Nieniewski and Zajączkowski (2017), who proposed a new metric for the evaluation of ultrasound (US) image filtering methods for the removal of speckle noise. Existing metrics are typically defined by comparing the original and filtered US images, which is, however, incorrect because no real US image is free of speckle noise and therefore the noisy original does not represent an appropriate comparison reference. To overcome these limitations, the authors propose to use the relative multivariate kurtosis, which measures the degree of deviation of a multivariate probability density from the multivariate normal distribution, and is evaluated within frames of filtered images containing edges. The proposed metric was evaluated against 16 different filtering methods on a simulated image, US images of cyst and blood vessel phantoms, and real US images of thyroid and carotid arteries. The results showed that visually more satisfactory filtering results were associated with a higher kurtosis, which may be also used to evaluate image quality. The main advantage of the proposed metric is that it is computed independently of any reference image, moreover, it is relatively consistent with visual perception and medical expert assessment.

\section{STEREOLOGY}

By identifying the lack of quantitative results in the specific field of brain myelin sheath analysis, $\mathrm{Li}$ et al. (2017) proposed to observe the problem from a stereological perspective. As existing studies have reported only qualitative but no quantitative evidence of degeneration and regeneration of myelin sheaths in aged brain white matter, they investigated both quantitative and qualitative changes by imaging the brain tissue of five young and five old female rats with transmission electron microscopy, and applying stereological techniques to the resulting photographs. After measuring the white matter volume, the volume density, total volume and thickness of myelin sheaths in the white matter, the length density, total length and diameter of myelinated fibers in the white matter, and tissue shrinkage in the white matter, a thorough statistical analysis of the obtained measurements was performed. According to the results, the study provided stereological evidence not only for the degeneration of myelin sheaths of smaller axons, but also for the regeneration of myelin sheaths in larger axons in aged brain white matter. The authors concluded that such age-dependent degeneration and regeneration of myelin sheaths may contribute to inducing a reduction in the velocity of conduction of action potentials, as well as a reduction in the agedependent perception.

In stereology, the nucleator is a form of geometric sampling for estimating the volume of a bounded object, and because of its efficiency and ease of use it is extensively applied in biosciences, especially for 
correct and efficient sampling and sizing of cells. For such a volume estimator, the intersection of the observed object with an isotropic random ray originating from a fixed pivotal point associated with the object has to be obtained, and the variance of the estimator can be reduced by increasing the number of rays. González-Villa et al. (2017) addressed the statistical performance of theoretical variance predictors for the nucleator by means of Monte Carlo resampling on computer reconstructions of a synthetic model and on magnetic resonance crosssections of a rat brain. Although the nucleator is a useful tool for estimating the volume, on the basis of the obtained results and their statistical analysis the authors concluded that the performance of the variance predictors was in general poor, which may be due to the inefficiency of the proposed design and the specific properties of the covariance function.

A similar topic was investigated by Kiderlen and Dorph-Petersen (2017), who focused on the Cavalieri's method for estimating the volume of a compact object from area measurements in equidistant parallel planar sections. In real applications, however, the section spacing and thickness can be quite irregular, and the classical Cavalieri estimator proved to be unbiased when the stack of parallel planes is stationary, but the variance is inflated and can be adjusted using variance approximations. In this study, the authors considered a special situation where the distances between consecutive section planes can be at least approximately measured. As a result, the Cavalieri estimator can be replaced by the trapezoidal or Simpson's quadrature rule with randomized, independently and identically perturbed sampling points, which was shown by a considerable variance reduction in the performed simulations. Moreover, the use of the trapezoidal rule was recommended as it is easier to implement than higher order quadrature rules and does not require any integrability condition for unbiasedness.

Gual-Arnau et al. (2017) proposed a numerical method for an invariant three-point curvature approximation for planar curves based on the arc of a parabolic sector, and analyzed the goodness of the proposed method against the true curvature and other approximation methods, namely the osculating circle and the angle-based method. The application of the method was first shown on an analytical example, and then on the problem of detecting cells in clusters, as observed in microscopic images. The results showed that by the proposed parabolic approach it is easier to detect the points of extreme curvature.

\section{The History of Stereology}

The editorial to the special topic "The History of Stereology" (Gądek-Moszczak, 2017) presented a short overview of the field of stereology. From the foundation of the International Society of Stereology - ISS in 1961 to its evolution to International Society for Stereology \& Image Analysis - ISSIA in 2016, the editorial summarized the activities through the fusion of stereology and image analysis, reflecting the response of stereology to the challenges of the times as a result of its multidisciplinary character. The first contribution to the special topic was an extensive and thorough historical survey of stereology by CruzOrive (2017), who in a chronological order presented in detail a series of topics that cover most aspects of stereology. From the geometrical probability and integral geometry, Cavalieri's principle, continuous and discrete sampling to the model-based and global stereology, individual particle sizing with size and orientation distributions, and efficiency estimation, the survey was concluded with a description of recent trends, indicating that advanced computing and image processing play an increasing role in classical stereology.

On the other hand, Vedel Jensen and Gundersen (2017) presented an overview of the biannual workshops on Stochastic Geometry, Stereology and Image Analysis - SGSIA that have been held every second year since 1981, with the last edition being therefore the $19^{\text {th }}$ and held for the first time in France. Through the workshop history along with the information of venues, organizers and participants, and a list of topics presented in workshop talks, the authors provided a concise description of the evolution and scientific impact of the workshops, and concluded that they will continue to be the main forum and occasion for communicating, discussing and promoting recent advances in stochastic geometry, spatial statistics, convex and integral geometry, stereology and image analysis.

The special topic was continued by Kubínová et al. (2017), who provided a historical overview of stereology as an unbiased methodological approach for the quantitative study of plant anatomy and cytology, with a particular focus on the evaluation of leaf structures with respect to the leaf being the most frequently studied plant organ. After providing a short history of the quantification of the plant anatomical structure, an overview of stereological methods applied in plant anatomy was given, focusing on different approaches to sampling, estimation of anatomical characteristics by plant surface area and volume density measurements, and plant cell ultrastructure measurements. In the 
discussion, other morphometric methods for the quantification of plant structures were presented and compared in terms of relevance, precision, unbiasedness and efficiency to stereological methods. In conclusion, the authors admitted that although stereology provides valuable an precise tools for plant structural measurements, its use and application have so far been relatively sparse. However, by broader publicity, development of dedicated software and incorporation of (semi)automated image analysis techniques, in their opinion stereology will find its way to the plant biology community as a powerful tool for investigating plant anatomical and cytological structures.

The final contribution to the special topic described the history of stereology in Poland from the beginning of the $20^{\text {th }}$ century up to the present. Gądek-Moszczak and Matusiewicz (2017) concentrated on the talented individuals and presented in detail their first significant steps in stereology and quantitative assessment of metal and rock structure that led to the creation of the Polish school of stereology, later formation of many scientific groups and centers for the application of stereology in metallography, start of the STERMAT series of conferences on stereology in materials in 1983, and ultimately to the foundation of the Polish Society for Stereology in 1988.

\section{STOHASTIC GEOMETRY}

From the perspective that random spatial patterns are the main topic of stohastic geometry, Beneš et al. (2017) studied the system of randomly dispersed segments on a plane with eventual interactions, and provided a biological application for the distribution of actin stress fibers in individual bone marrow stem cells as observed by fluorescence imaging. By mathematical modeling with a finite point process of a density with respect to the Poisson process, a homogeneous and an inhomogeneous model was introduced. For both models, the parameters were obtained by the TakacsFiksel estimator, and the degree of fit was evaluated by Monte Carlo experiments, yielding satisfactory results. However, the homogeneous model proved to be more adequate for simulated data, while the inhomogeneous model proved valuable for real data, which was confirmed by the described biological application.

Often used in microscopy and stereology, systematic sampling is a statistical method that selects samples from a larger population according to a random starting point and a fixed periodic interval. In $3 \mathrm{D}$, a stack of parallel and equally spaced plane sections through the spatial structure of interest is generated, and then observations are usually made at a regular lattice of grid points within each section.
However, real sampling procedures may involve technically very challenging physical cutting, e.g., the generation of ultra thin sections for electron microscopy where the distance between adjacent sections is in the order of nanometers. In such cases, the actual section distance may deviate from the intended distance. Rønn-Nielsen et al. (2017) studied the situation where a stationary and isotropic random field is observed on two parallel planes with unknown distance, and evaluated the approximate variance of an existing estimator for the distance between the two planes under the class of Lévy random fields. By describing two different simulation studies, the authors concluded that the applied distance estimator as well as the variance approximation method perform well.

\section{CONCLUSION}

The overview of contributions published in IAS volume 36 (2017) revealed that the readership was offered with a large variety of topics within the broader field of stereology and image analysis, which were enriched by the review papers, united under the special topic "The History of Stereology". It can be therefore concluded that the contributions reflect the scope of the IAS journal that is guided by the multidisciplinary fusion of stereology and image analysis.

\section{REFERENCES}

Baran M, Tabor Z (2017). Principal geodesic analysis boundary delineation with superpixel-based constraints. Image Anal Stereol 36:223-32.

Bawa VS, Kumar K, Kumar V (2017). A generalized nonlinear method for distortion correction and top-down view conversion of fish eye images. Image Anal Stereol 36:141-50.

Beneš V, Večeřa J, Eltzner B, Wollnik C, Rehfeldt F, Králová $\mathrm{V}$, Huckemann S (2017). Estimation of parameters in a planar segment process with a biological application. Image Anal Stereol 36:25-33.

Cruz-Orive LM (2017). Stereology: a historical survey. Image Anal Stereol 36:153-77.

Gądek-Moszczak A (2017). History of stereology. Image Anal Stereol 36:151-2.

Gądek-Moszczak A, Matusiewicz P (2017). Polish stereology - a historical review. Image Anal Stereol 36:207-21.

González-Villa J, Cruz M, Cruz-Orive LM (2017). On the precision of the nucleator. Image Anal Stereol 36:12132. 
Gual-Arnau X, Ibáñez MV, Monterde J (2017). Curvature approximation from parabolic sectors. Image Anal Stereol 36:233-41.

Jouannot-Chesney P, Jernot JP, Lantuéjoul C (2017). Percolation transition and topology. Image Anal Stereol 36:95-103.

Kartbaev T, Akhmetov B, Doszhanova A, Mukapil K, Kalizhanova A, Nabiyeva G, Balgabayeva L, Malikova F (2017). Development of a computer system for identity authentication using artificial neural networks. Image Anal Stereol 36:51-64.

Khordehchi AE, Ayatollahi A, Daliri MR (2017). Automatic lung nodule detection based on statistical region merging and support vector machines. Image Anal Stereol 36:65-78.

Kiderlen M, Dorph-Petersen KA (2017). The Cavalieri estimator with unequal section spacing revisited. Image Anal Stereol 36:133-9.

Koçak C. CLSM: couple layered security model a highcapacity data hiding scheme using with steganography. Image Anal Stereol 36:15-23.

Kreft M (2017). Research highlights in IAS. Image Anal Stereol 36:1-3.

Kubínová L, Radochová B, Lhotáková Z, Kubínová Z, Albrechtová J (2017). Stereology, an unbiased methodological approach to study plant anatomy and cytology: past, present and future. Image Anal Stereol
36:187-205.

Li C, Zhang L, Ma Q, Tang Y, He Y (2017). Stereological evidence for de/re-generation of myelin sheaths in aged brain white matter of female rats. Image Anal Stereol 36:111-20.

Niedźwiecki M, Tomaszuk M, Kabat D, Latałla Z, Rzecki K, Tabor Z (2017). Application of image analysis methods for isocenter quality assurance in radiotherapy. Image Anal Stereol 36:35-41.

Nieniewski M, Zajączkowski P (2017). Comparison of ultrasound image filtering methods by means of multivariable kurtosis. Image Anal Stereol 36:79-94.

Rønn-Nielsen A, Sporring J, Vedel Jensen EB (2017). Estimation of sample spacing in stochastic processes. Image Anal Stereol 36:43-9.

Šedivý O, Dake JM, Krill CE III, Schmidt V, Jäger A (2017). Description of 3D morphology of grain boundaries in aluminum alloys using tessellation models generated by ellipsoids. Image Anal Stereol 36:5-13.

Vedel Jensen EB, Gundersen HJG (2017). The workshops on stochastic geometry, stereology and image analysis. Image Anal Stereol 36:179-85.

Wejrzanowski T, Ibrahim SH, Skibinski J, Cwieka K, Kurzydlowski KJ (2017). Appropriate models for simulating open-porous materials. Image Anal Stereol 36:105-10. 